\title{
Incomplete penetrance
}

National Cancer Institute ( $\mathrm{NCl})$

\section{Source}

National Cancer Institute (NCI). Incomplete penetrance.

Penetrance refers to the likelihood that a clinical condition will occur when a particular genotype is present. A condition is said to show incomplete penetrance when some individuals who carry the pathogenic variant express the associated trait while others do not. 